AdVANCES IN COMBINATORICS, 2021:2, 8 pp.

www.advancesincombinatorics.com

\title{
On Partitioning the Edges of an Infinite Digraph into Directed Cycles
}

\author{
Attila Joó * \\ Received 15 October 2018; Revised 10 July 2020; Published 15 January 2021
}

\begin{abstract}
Nash-Williams proved in [1] that for an undirected graph $G$ the set $E(G)$ can be partitioned into cycles if and only if there is no finite cut of odd size. Later $\mathrm{C}$. Thomassen gave a simpler proof for this in [2] and conjectured the following directed analogue of the theorem: the edge set of a digraph can be partitioned into directed cycles if and only if for each subset of the vertices the cardinality of the ingoing and the outgoing edges are equal. The aim of the paper is to prove this conjecture.
\end{abstract}

Key words and phrases: infinite digraph, directed cycle, edge-partition

\section{Introduction}

One of Nash-Williams' famous results in infinite graph theory is the following:

Theorem 1 (Nash-Williams, [1] (p. 235 Theorem 3)). If $G$ is an undirected graph, then $E(G)$ can be partitioned into cycles if and only if there is no finite cut of odd size.

After giving a simpler proof for Theorem 1, C. Thomassen conjectured a directed version of it (see [2] p. 1037). Since the main result of our paper is deciding this conjecture positively, we state it as a theorem.

Theorem 2. If $D=(V, E)$ is a directed graph, then $E$ can be partitioned into directed cycles if and only if for all $X \subseteq V$ the cardinalities of the set of the ingoing and the outgoing edges of $X$ are equal.

*Funding was provided by the Alexander von Humboldt Foundation and by NKFIH OTKA-129211. 
It is worth to mention that already Nash-Williams himself claimed Theorem 2 to be true in [1]. L. Soukup gave a new shorter proof for Theorem 1 (see Theorem 5.1 of [3]) based on elementary submodels. The main difficulty of the proof of Theorem 2 compared to the undirected variant is the following. The obstacle for the cycle partition in Theorem 1 is a finite set (an odd cut) but the obstacle usually fails to be finite in the context of Theorem 2.

The paper is designed to be comprehensible for everybody with a basic familiarity in infinite combinatorics. No advanced set theoretic concepts are used. Our main tool is the elementary submodel method and although it is relatively common in this field, we introduce it briefly. A more detailed introduction to elementary submodels where not even basic logic background (first order formulas and models) is assumed can be found in [3] with several applications in infinite combinatorics including a proof of Theorem 1.

\section{Notation}

The digraphs $D=(V, E)$ in the paper may have loops and parallel edges. ${ }^{1}$ For a subset $X$ of $V$, we denote by out ${ }_{D}(X)$ and $\operatorname{in}_{D}(X)$ the set of outgoing and ingoing edges of $X$ in $D$ respectively ${ }^{2}$ and let cut $_{D}(X):=$ out $_{D}(X) \cup \operatorname{in}_{D}(X)$. For an $X \subseteq V$ let $\boldsymbol{D}[X]$ be the subgraph of $D$ induced by $X$. The weak components of a digraph are the components of its underlying undirected graph. We call a digraph weakly connected if it has just one weak component, i.e., its undirected underlying graph is connected. If $x, y$ are vertices of the path $P$, then we denote by $\boldsymbol{P}[x, y]$ the segment of $P$ between $x$ and $y$ (including $x$ and $y$ ). For an undirected graph $G$ and $u \neq v \in V(G)$, we write $\lambda_{G}(\boldsymbol{u}, \boldsymbol{v})$ for the local edge-connectivity between $u$ and $v$ in $G$, i.e., the smallest cardinal $\kappa$ such that it is possible to delete $\kappa$ many edges in such a way that $u$ and $v$ are in different components of the resulting graph. Let us recall that the local edge-connectivity is also the maximal cardinal $\kappa$ such that there is a system $\mathcal{P}$ of pairwise edge-disjoint paths of size $\kappa$ between $u$ and $v$. We call a subset $X$ of $V$ overloaded (with respect to $D$ ) if $\left|\operatorname{out}_{D}(X)\right|<\left|\operatorname{in}_{D}(X)\right|$. A digraph $D$ is called unbalanced if it admits an overloaded vertex set and $D$ is balanced if it is not unbalanced.

The variables $\boldsymbol{\alpha}$ and $\boldsymbol{\beta}$ are standing always for ordinal numbers while we use $\boldsymbol{\kappa}$ and $\boldsymbol{\lambda}$ for cardinals.

\section{Elementary submodels and basic facts}

We give here a quick overview about elementary submodel techniques which play a central role in our proof. One can find a more detailed introduction with many combinatorial applications in [3].

Roughly speaking, elementary submodels are sets which are closed under all possible (relevant) operations. Instead of ensuring the desired closures "by hand" it provides a flexible uniform framework giving effortlessly all the closures we want to use. To make this precise, we need to apply some basic model theoretic concepts. All the formulas and models in this paper are in the first order language of set theory and the models are $\in$-models, i.e., the "element of" relation in them is the real " $\in$ ". Let $\Sigma=\left\{\varphi_{1}, \ldots, \varphi_{n}\right\}$ be a finite set of formulas where the free variables of $\varphi_{i}$ are $x_{i, 1}, \ldots, x_{i, n_{i}}$. We call a set

\footnotetext{
${ }^{1}$ One can reduce Theorem 2 to the case of simple digraphs by the subdivision of parallel edges with a new vertex together with the deletion of loops but it would not make any difference in our proof.

${ }^{2}$ Edge $e$ is ingoing with respect to $X$ if its head is in $X$ but its tail is not.
} 
$M$ a $\Sigma$-elementary submodel if $|M| \subseteq M$ holds $^{3}$ and the formulas in $\Sigma$ are absolute between $M$ and the universe, i.e.,

$$
\bigwedge_{i=1}^{n}\left[\forall x_{1}, \ldots, x_{n_{i}} \in M\left[\left(M \models \varphi_{i}\left(x_{1}, \ldots, x_{n_{i}}\right)\right) \Longleftrightarrow \varphi_{i}\left(x_{1}, \ldots, x_{n_{i}}\right)\right]\right]
$$

A fundamental fact we need (Corollary 2.6 in [3]) that one can find elementary submodels with certain prescribed parameters:

Proposition 3. For every infinite cardinal $\kappa$, every finite set $\Sigma$ of formulas and every set $x$ there exists a $\Sigma$-elementary submodel $M$ of size $\kappa$ with $x \in M$.

Defining a concrete $\Sigma$ in a particular application is usually pointless. The common practise is "pretending" that $\Sigma$ contains always what we need. These instances define implicitly afterwards what the minimal sufficient $\Sigma$ is. Every $\Sigma$-elementary submodel is closed under all operations $F\left(x_{1}, \ldots, x_{n}\right)=y$ defined by a formula $\varphi\left(x_{1}, \ldots, x_{n}, y\right)$ for which

$$
\forall x_{1} \ldots \forall x_{n} \exists ! y \varphi\left(x_{1}, \ldots, x_{n}, y\right), \varphi\left(x_{1}, \ldots, x_{n}, y\right) \in \Sigma \text {. }
$$

Indeed, the first formula ensures that for every $a_{1}, \ldots, a_{n} \in M$ there is a $b \in M$ such that $M=$ $\varphi\left(a_{1}, \ldots, a_{n}, b\right)$ while the second guarantees that $F\left(a_{1}, \ldots, a_{n}\right)=b$. To give a more explicit example, if a digraph $D$ and one of its edges $e$ are in the $\Sigma$-elementary submodel $M$, then the endpoints of $e$ are also in $M$ because they are definable from $D$ and $e$ and the corresponding defining formulas are assumed to be in $\Sigma$. From now on whenever we claim that $\Sigma$-elementary submodels have some specific properties, it is meant under the assumptions that $\Sigma$ contains the necessary formulas.

To illustrate arguments involving elementary submodels, we prove now three statements (which we need later anyway). Because of the introductory nature of this section we give more details in the proofs about how absoluteness is used and with which formulas than it is usual in the normal practise. In order to keep the arguments short, we will put some formulas "redundantly" in $\Sigma$ instead of using the fact that they are absolute even without being in $\Sigma$.

Proposition 4. If $M$ is a $\Sigma$-elementary submodel and $X \in M$ with $|X| \leq|M|$, then $X \subseteq M$.

Proof. We assume that there are formulas in $\Sigma$ expressing the following:

1. For every $X$ there is a cardinal $\kappa$ such that $|X|=\kappa$.

2. $|X|=\kappa$.

3. $f$ is a bijection between $X$ and $Y$.

4. $f$ is a function and $f(x)=y$.

${ }^{3}$ The condition $|M| \subseteq M$ is not always included in the definition of elementary submodels but it is a convenient assumption. 
Since $X \in M$ and formula 1 is in $\Sigma$ there is a $\kappa \in M$ such that $M \models|X|=\kappa$. Because of formula 2 is in $\Sigma$, $|X|=\kappa$ must hold. Then $M=$ " $\exists$ bijection from $\kappa$ to $X$ " and therefore by basic logic there is some $f \in M$ such that $M=$ " $f$ is a bijection from $\kappa$ to $X$ ". As earlier, it must be true in the universe because formula 3 is in $\Sigma$. It follows by basic logic that for every $\alpha \in M \cap \kappa$, there is a $y_{\alpha} \in M$ with $M=f(\alpha)=y_{\alpha}$. Since formula 4 is in $\Sigma, f(\alpha)=y_{\alpha}$ holds for $\alpha \in M \cap \kappa$. But $\kappa=|X| \subseteq|M| \subseteq M$ where the last inclusion was built in the definition of elementary submodels. Therefore $M \cap \kappa=\kappa$ and hence $f(\alpha) \in M$ for every $\alpha<\kappa$ from which $X \subseteq M$ follows because $f: \kappa \rightarrow X$ is a bijection.

If $M$ is a $\Sigma$-elementary submodel containing the directed or undirected graph $G=(V, E)$, then we define $\boldsymbol{G}(\boldsymbol{M}):=(V \cap M, E \cap M)$ and $\boldsymbol{G} \backslash \boldsymbol{M}:=(V, E \backslash M)$ which are subgraphs of $G$.

Proposition 5. Let $G$ be an undirected graph an let $M$ be a $\Sigma$-elementary submodel with $G \in M$. Assume that $\lambda_{G \backslash M}(u, v)>0$ for some $u \neq v \in V(G) \cap M$. Then $\lambda_{G}(u, v)>|M|$.

Proof. We assume that $\Sigma$ contains the formulas that expressing the following:

1. $(\forall G)(\forall u \neq v \in V(G)) \exists \kappa\left(\lambda_{G}(u, v)=\kappa\right)$.

2. $\lambda_{G}(u, v)=\kappa$.

3. $E^{\prime} \subseteq E(G)$ separates the vertices $u$ and $v$ in graph $G$.

4. $|X|=\kappa$.

Let $u \neq v \in V(G) \cap M$ be arbitrary and suppose that $\lambda_{G}(u, v)=: \kappa \leq|M|$. We have to show that $\lambda_{G \backslash M}(u, v)=0$. Since $G, u, v \in M$ and $\kappa$ is definable from them and the formulas 1 and 2 are in $\Sigma$, we know that $\kappa \in M$ and $M=\lambda_{G}(u, v)=\kappa$. Then there is some $E^{\prime}$ such that $M=$ " $E^{\prime} \subseteq E(G)$ separates the vertices $u$ and $v$ in graph $G$ and $\left|E^{\prime}\right|=\kappa^{\prime \prime}$. Formula 3 ensures that $E^{\prime} \subseteq E(G)$ separates the vertices $u$ and $v$ in graph $G$ and formula 4 guarantees that $\left|E^{\prime}\right|=\kappa$. But then by Proposition $4, E^{\prime} \subseteq M$ and therefore $\lambda_{G \Downarrow M}(u, v)=0$.

We need the following result of L. Soukup (see [3] Lemma 5.3 on p. 16):

Proposition 6. Let $G$ be an undirected graph and let $M$ be a $\Sigma$-elementary submodel with $G \in M$. Assume that $x \neq y \in V(G)$ are in the same component of $G \backslash M$ and $F \subseteq E(G \rrbracket M)$ separates them where $|F| \leq|M|$. Then $F$ separates $x$ and $y$ in the whole $G$.

Proof. Assume (reductio ad absurdum) that it is false and $G, F, x, y, M$ witness it. We take a path $P$ between $x$ and $y$ in $G \backslash F$. Let $x^{\prime}$ and $y^{\prime}$ be the first and the last intersection of $P$ with $V(G) \cap M$ with respect to some direction of $P$. The vertices $x^{\prime}$ and $y^{\prime}$ are well-defined and distinct since $P$ necessarily uses some edge from $E(G) \cap M$. We also fix a path $Q$ between $x$ and $y$ in $G \backslash M$. The paths $P\left[x^{\prime}, x\right], Q, P\left[y, y^{\prime}\right]$ shows that $x^{\prime}$ and $y^{\prime}$ are in the same component of $G \backslash M$. Thus by Proposition 5, $\lambda_{G}\left(x^{\prime}, y^{\prime}\right)>|M|$. There is a path $R$ between $x^{\prime}$ and $y^{\prime}$ in $G \rrbracket F$ since $\lambda_{G}\left(x^{\prime}, y^{\prime}\right)>|M| \geq|F|$. But then $P\left[x, x^{\prime}\right], R, P\left[y^{\prime}, y\right]$ shows that $F$ does not separate $x$ and $y$ in $G \backslash M$ which is a contradiction. 


\section{On Partitioning the Edges of An Infinite DigRaph into Directed Cycles}

\section{Proof of the main result}

Proof of Theorem 2. In any digraph $D=(V, E)$ for every $X \subseteq V$ the contribution of a directed cycle to $\left|\operatorname{in}_{D}(X)\right|$ and $\mid$ out $_{D}(X) \mid$ is the same, thus if $E$ can be partitioned into directed cycles, then $D$ must be balanced.

For countable digraphs the other direction of the equivalence is also easy. Let $D=(V, E)$ be a balanced countable digraph. Observe that for each weak component $X, D[X]$ must be strongly connected. Thus every $e \in E$ is in some directed cycle of $D$. Note that, a balanced digraph remains balanced after the deletion of the edges of a directed cycle. We create a desired partition by recursion. Let $<$ be an $|E|$-type ordering of $E$ and $D_{0}:=D$. In the $n$-th step we take a directed cycle $C_{n}$ in $D_{n}$ through its $<$-smallest edge and define $D_{n+1}:=D_{n} \| E\left(C_{n}\right)$. Clearly, the resulting cycles $C_{n}$ give a desired partition.

For uncountable digraphs the analogue of this naive recursive approach does not work because in a transfinite recursion one cannot ensure that after the first limit step the remaining digraph is still balanced.

Lemma 7. For every infinite cardinal $\kappa$ and every set $x$ there is a $\Sigma$-elementary submodel $M$ of size $\kappa$ with $x \in M$ such that for any balanced digraph $D \in M$ the edge set $E(D) \cap M$ can be partitioned into directed cycles.

Theorem 2 follows directly from Lemma 7: let $D=(V, E)$ be an arbitrary balanced digraph and we use Lemma 7 with $x:=D$ and $\kappa:=|E|+\boldsymbol{\aleph}_{0}$. Then Proposition 4 guarantees $E \subseteq M$ which ensures $D=D(M)$. Hence Lemma 7 gives a desired partition for $D$ itself.

Proof. We prove Lemma 7 by transfinite induction on $\kappa$. Consider first the case $\kappa=\boldsymbol{\aleph}_{0}$. Let $M$ be an arbitrary countable $\Sigma$-elementary submodel with $x \in M$ (such an $M$ exists by Proposition 3). Assume that $D=(V, E) \in M$ is a digraph such that $E \cap M$ cannot be partitioned into directed cycles. We have to show that $D$ is unbalanced. We know that $D(M)$ must be unbalanced because it is countable and we have already proved Theorem 2 for countable digraphs. Let $X \subseteq V \cap M$ be an overloaded set in $D(M)$. Then out $_{D(M)}(X)$ is finite because

$$
\mid \text { out }_{D(M)}(X)|<| \operatorname{in}_{D(M)}(X)|\leq| M \mid=\aleph_{0} .
$$

Let $S$ be a set whose elements are the tails of the edges in $\operatorname{out}_{D(M)}(X)$ and the heads of at least $\mid$ out $_{D(M)}(X) \mid+1$ many edges from $\operatorname{in}_{D(M)}(X)$. Consider the set $Y$ of vertices that are reachable by a directed path from $S$ in $D$ without using any edges from out ${ }_{D(M)}(X)$. We show that $Y$ is overloaded in $D$. It follows directly from the definition of $Y$ that out $D(Y) \subseteq$ out $_{D(M)}(X)$. In order to show that $\left|\operatorname{in}_{D}(Y)\right| \geq \mid$ out $_{D(M)}(X) \mid+1$, let an $e \in \operatorname{in}_{D(M)}(X)$ with its head in $S$ be fixed. To guarantee that $e \in \operatorname{in}_{D}(Y)$, we need to show that the tail of $e$ is not in $Y$, i.e., it is not reachable from $S$ in $D$ without using edges from out $_{D(M)}(X)$. Suppose for a contradiction that it is. Then by $D, S, e$, out $_{D(M)}(X) \in M$ there is a directed path $P \in M$ witnessing this. But then by Proposition $4, E(P) \subseteq M$ and hence $P$ lies in $D(M)$. Since $P$ starts in $X$ but terminates out of $X$ it must use some edge from out $D(M)(X)$ which is a contradiction. Since there are at least $\mid$ out $_{D(M)}(X) \mid+1$ such an edge $e,\left|\operatorname{in}_{D}(Y)\right| \geq \mid$ out $_{D(M)}(X) \mid+1$ follows. We can conclude that $Y$ is an overloaded set in $D$ and thus $D$ is unbalanced.

Let $\lambda>\boldsymbol{\aleph}_{0}$ and assume that Lemma 7 is true for $\kappa<\lambda$. We define a sequence of $\Sigma$-elementary submodels $\left\langle M_{\alpha}: \alpha<\lambda\right\rangle$ by transfinite recursion such that for all $\alpha<\lambda$ : 
1. $x \in M_{\alpha}$,

2. $\left|M_{\alpha}\right|=|\alpha|+\boldsymbol{\kappa}_{0}$,

3. $\alpha, M_{\alpha} \in M_{\alpha+1}$,

4. if $D \in M_{\alpha+1}$ is a balanced digraph, then the edge-set of $D\left(M_{\alpha+1}\right)$ (i.e. $\left.E \cap M_{\alpha+1}\right)$ can be partitioned into directed cycles,

5. $M_{\alpha}=\bigcup_{\beta<\alpha} M_{\beta}$ if $\alpha$ is a limit ordinal.

Note that Proposition 4 guarantees that $M_{\beta} \subseteq M_{\alpha}$ for $\beta<\alpha<\lambda$. Let $M_{0}$ be an arbitrary countable $\Sigma$-elementary submodel containing $x$. Suppose that $M_{\beta}$ is already defined if $\beta<\alpha$ for some $\alpha<\lambda$ and satisfies the properties above. If $\alpha$ is a limit ordinal, then our only choice is $M_{\alpha}:=\bigcup\left\{M_{\beta}: \beta<\alpha\right\}$. Then $M_{\alpha}$ is a $\Sigma$-elementary submodel since it is the increasing union of $\Sigma$-elementary submodels ${ }^{4}$. If $\alpha=\beta+1$, then we apply the induction hypothesis with cardinal $|\alpha|+\boldsymbol{\aleph}_{0}<\lambda$ and set $\left\{\beta, M_{\beta}\right\}$ to obtain an $M_{\beta+1}$ satisfying the conditions. The recursion is done.

Let $M:=\bigcup\left\{M_{\alpha}: \alpha<\lambda\right\}$. Then $M$ is a $\Sigma$-elementary submodel of size $\lambda$ and $x \in M$. Let $D \in M$ be a balanced digraph and let $\beta+1<\lambda$ be the smallest ordinal such that $D \in M_{\beta+1}$. We define $D_{\beta}$ to be $D\left(M_{\beta+1}\right)$ and for $\alpha$ with $\beta<\alpha<\lambda$ let $D_{\alpha}:=\left(D \| M_{\alpha}\right)\left(M_{\alpha+1}\right)$. These are edge-disjoint subgraphs of $D(M)$, moreover, $\left\{E\left(D_{\alpha}\right): \beta \leq \alpha<\lambda\right\}$ is a partition of $E(D) \cap M$. Since $\left(D \Downarrow M_{\alpha}\right)$ is definable from $D, M_{\alpha} \in M_{\alpha+1}$, we have $\left(D \Downarrow M_{\alpha}\right) \in M_{\alpha+1}$.

Claim 8. If $M$ is a $\Sigma$-elementary submodel and $D \in M$ is a balanced digraph, then $D \rrbracket M$ is also balanced.

If we prove Claim 8, then we are done with the proof of Lemma 7 as well. Indeed, by Claim 8, the digraphs $D \backslash M_{\alpha}$ are balanced and therefore by using property 4 with $D \| M_{\alpha}$ and $M_{\alpha+1}$ we can partition $E\left(D_{\alpha}\right)$ into directed cycles for all $\alpha$ with $\beta \leq \alpha<\lambda$. By uniting these partitions, we obtain a desired partition of $E \cap M$.

Before we turn to the proof of Claim 8, we need the following observation to find overloaded sets in an unbalanced digraph with an extra property.

Proposition 9. If $D=(V, E)$ is an unbalanced digraph, then it has a weak component $Z$ and a partition $Z=X \cup Y$ such that $D[X]$ and $D[Y]$ are weakly connected and $X$ is overloaded.

Proof. Let $X^{\prime} \subseteq V$ be overloaded and let $X_{i}(i \in I)$ be the weak components of $D\left[X^{\prime}\right]$. Then

$$
\sum_{i \in I}\left|\operatorname{out}_{D}\left(X_{i}\right)\right|=\left|\operatorname{out}_{D}\left(X^{\prime}\right)\right|<\left|\operatorname{in}_{D}\left(X^{\prime}\right)\right|=\sum_{i \in I}\left|\operatorname{in}_{D}\left(X_{i}\right)\right|
$$

and therefore there is an $i_{0} \in I$ such that $\left|\operatorname{out}_{D}\left(X_{i_{0}}\right)\right|<\left|\operatorname{in}_{D}\left(X_{i_{0}}\right)\right|$. Let $Z$ be the weak component of $D$ that contains $X_{i_{0}}$ and let $Y_{j}(j \in J)$ be the weak components of $D\left[Z \backslash X_{i_{0}}\right]$. Then

$$
\sum_{j \in J}\left|\operatorname{in}_{D}\left(Y_{j}\right)\right|=\left|\operatorname{out}_{D}\left(X_{i_{0}}\right)\right|<\left|\operatorname{in}_{D}\left(X_{i_{0}}\right)\right|=\sum_{i \in J}\left|\operatorname{out}_{D}\left(Y_{j}\right)\right|
$$

${ }^{4}$ This implication is a basic fact from model theory, the proof is a straightforward formula induction. 
and thus there is a $j_{0} \in J$ such that $\left|\operatorname{in}_{D}\left(Y_{j_{0}}\right)\right|<\mid$ out $_{D}\left(Y_{j_{0}}\right) \mid$. Let $Y:=Y_{j_{0}}$ and $X:=Z \backslash Y_{j_{0}}$. Then $X$ is overloaded and both $X=X_{i_{0}} \cup\left\{Y_{j}: j \in J \backslash\left\{j_{0}\right\}\right\}$ and $Y=Y_{j_{0}}$ induce a weakly connected subdigraph in $D$ because of the definition of the sets $Y_{j}(j \in J)$.

Proof of Claim 8. Assume for contradiction, that $D \rrbracket M$ is unbalanced. Then by Proposition 9 there is a weak component $Z$ of $D \backslash M$ with a partition $Z=X \cup Y$ such that $(D \Downarrow M)[X]$ and $(D \rrbracket M)[Y]$ are weakly connected and $X$ is overloaded in $D \rrbracket M$. Let $F:=\operatorname{cut}_{D \Downarrow M}(X)$. Next we show that $|F| \leq|M|$. We may suppose that $F$ is infinite and thus $\operatorname{cut}_{D}(X)$ as well since $F \subseteq \operatorname{cut}_{D}(X)$. Thus $\boldsymbol{\aleph}_{0} \leq\left|\operatorname{out}_{D}(X)\right|=\left|\mathrm{in}_{D}(X)\right|$. We must have $\mid$ out $_{D \Downarrow M}(X) \mid<$ out $_{D}(X) \mid$ since otherwise

$$
\left|\operatorname{out}_{D \Downarrow M}(X)\right|=\left|\operatorname{out}_{D}(X)\right|=\left|\operatorname{in}_{D}(X)\right| \geq\left|\operatorname{in}_{D \Downarrow M}(X)\right|
$$

which contradicts the choice of $X$. Hence $M$ contains $\left|\mathrm{out}_{D}(X)\right|$ elements of $\operatorname{out}_{D}(X)$ and thus $\mid$ out $_{D}(X)|\leq| M \mid$. Then

$$
|F|=\left|\operatorname{in}_{D \Downarrow M}(X)\right|+\left|\operatorname{out}_{D \Downarrow M}(X)\right| \leq\left|\operatorname{in}_{D}(X)\right|+\operatorname{out}_{D}(X)|=| \text { out }_{D}(X)|\leq| M \mid .
$$

By using Proposition 6 to the undirected underlying graph of $D$ with $F$ and with arbitrary $x \in X$ and $y \in Y$, we conclude that $X$ and $Y$ belong to distinct weak components of $D \rrbracket F$. Let us denote by $X^{\prime}$ and $Y^{\prime}$ these components respectively. We claim that $\operatorname{cut}_{D}\left(X^{\prime}\right)=F$. Indeed, cut $_{D}\left(X^{\prime}\right) \subseteq F$ follows directly from the definition of $X^{\prime}$, furthermore, the elements of $F$ go between $X$ and $Y$ and therefore between $X^{\prime}$ and $Y^{\prime}$. But then $\mid$ out $_{D \Downarrow M}(X)|=|$ out $_{D}\left(X^{\prime}\right) \mid$ and $\left|\operatorname{in}_{D \Downarrow M}(X)\right|=\left|\operatorname{in}_{D}\left(X^{\prime}\right)\right|$ thus

$$
\left|\operatorname{out}_{D}\left(X^{\prime}\right)\right|=\left|\operatorname{out}_{D \Downarrow M}(X)\right|<\left|\operatorname{in}_{D \Downarrow M}(X)\right|=\left|\operatorname{in}_{D}\left(X^{\prime}\right)\right|
$$

therefore $X^{\prime}$ is overloaded in $D$ which is a contradiction.

\section{References}

[1] Nash-Williams, C. S. J. Decomposition of graphs into closed and endless chains. Proceedings of the London Mathematical Society 3, 1 (1960), 221-238. 1, 2

[2] Thomassen, C. Nash-williams' cycle-decomposition theorem. Combinatorica 37, 5 (2017), 1027-1037. 1

[3] Soukup, L. Elementary submodels in infinite combinatorics. Discrete Mathematics 311, 15 (2011), 1585-1598. 2, 3, 4 
ATtila Joó

\section{AUTHOR}

Attila Joó

University of Hamburg and Egerváry Research Group on Combinatorial Optimization Hamburg, Germany

attila.joo@uni-hamburg.de

https://www.math.uni-hamburg.de/home/joo 